\title{
Exercise modulates postreceptor insulin signaling and glucose transport in muscle-specific insulin receptor knockout mice
}

\author{
Jørgen F.P. Wojtaszewski, Yasuki Higaki, Michael F. Hirshman, M. Dodson Michael, \\ Scott D. Dufresne, C. Ronald Kahn, and Laurie J. Goodyear
}

Research Division, Joslin Diabetes Center and Department of Medicine, Brigham and Women's Hospital and Harvard Medical School, Boston, Massachusetts 02215, USA

Address correspondence to: Laurie J. Goodyear, Joslin Diabetes Center, One Joslin Place, Boston, Massachusetts 02215, USA.

Phone: (617) 732-2573; Fax: (617) 732-2650; E-mail: laurie.goodyear@joslin.harvard.edu.

Received for publication July 26, 1999, and accepted in revised form September 22, 1999.

Physical exercise promotes glucose uptake into skeletal muscle and makes the working muscles more sensitive to insulin. To understand the role of insulin receptor (IR) signaling in these responses, we studied the effects of exercise and insulin on skeletal muscle glucose metabolism and insulin signaling in mice lacking insulin receptors specifically in muscle. Muscle-specific insulin receptor knockout (MIRKO) mice had normal resting 2-deoxy-glucose (2DG) uptake in soleus muscles but had no significant response to insulin. Despite this, MIRKO mice displayed normal exercise-stimulated 2DG uptake and a normal synergistic activation of muscle 2DG uptake with the combination of exercise plus insulin. Glycogen content and glycogen synthase activity in resting muscle were normal in MIRKO mice, and exercise, but not insulin, increased glycogen synthase activity. Insulin, exercise, and the combination of exercise plus insulin did not increase IR tyrosine phosphorylation or phosphatidylinositol 3-kinase activity in MIRKO muscle. In contrast, insulin alone produced a small activation of Akt and glycogen synthase kinase-3 in MIRKO mice, and prior exercise markedly enhanced this insulin effect. In conclusion, normal expression of muscle insulin receptors is not needed for the exercise-mediated increase in glucose uptake and glycogen synthase activity in vivo. The synergistic activation of glucose transport with exercise plus insulin is retained in MIRKO mice, suggesting a phenomenon mediated by nonmuscle cells or by downstream signaling events.

J. Clin. Invest. 104:1257-1264 (1999).

\section{Introduction}

Insulin action in skeletal muscle is mediated by receptor binding at the cell surface of the muscle fibers. This initiates a complex series of intracellular signaling events that lead to the numerous cellular effects of insulin including increased glucose uptake and glycogen synthesis (reviewed in refs. 1 and 2). Physical exercise also plays an important role in skeletal muscle glucose and glycogen metabolism $(3,4)$; however, the intracellular mechanisms initiating these actions of contractile activity are poorly understood. Contraction of isolated skeletal muscle in vitro can increase glucose transport in the absence of insulin, suggesting that these stimuli use distinct mechanisms for the activation of glucose transport (5-7). On the other hand, in exercising rats $(8)$, dogs $(9,10)$, and humans $(11,12)$, full activation of glucose uptake appears to require simultaneous exposure of the muscle to insulin. In fact, the presence of very low levels of circulating insulin profoundly influences skeletal muscle glucose uptake during exercise in dogs (10). Therefore, it is still not clear whether the increase in muscle glucose uptake in response to exercise in vivo can occur independent of insulin action.
The hypothesis that different mechanisms are involved in exercise- and insulin-stimulated glucose transport in skeletal muscle is supported by data showing that these 2 stimuli have additive effects when applied simultaneously $(7,13,14)$. There are also some circumstances in which the combination of exercise plus insulin can have an even greater effect on glucose uptake than would be predicted from the sum of the effects of each stimulus alone - i.e., a "synergistic effect" $(8-13,15,16)$. It is also well established that skeletal muscle displays increased insulin sensitivity for glucose transport and glycogen synthesis for a prolonged period after contractile activity has ceased (reviewed in refs. 3,17 , and 18). Taken together, these findings suggest that under some conditions, exercise and insulin elicit crosstalk between the signaling molecules that mediate these responses in muscle. One potential mechanism for this increase in insulin action could involve the ability of exercise to enhance insulin signaling, although studies in this area have been inconsistent. For example, insulin signaling at the level of insulin receptor substrate-1-associated (IRS-1-associated) phosphatidylinositol 3-kinase (PI 3-kinase) activity is reduced in rat hind-limb skeletal muscle after contractions in situ (19), 


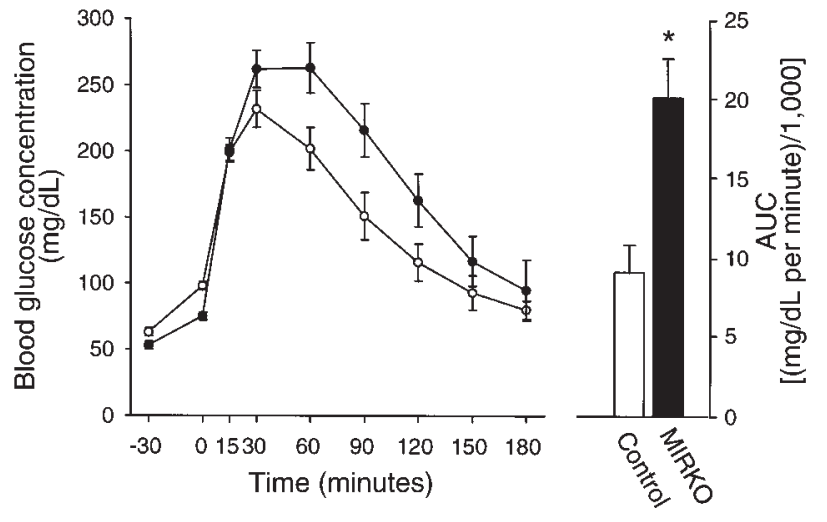

Figure 1

Glucose tolerance test. Basal glucose concentrations were measured in blood taken from the tail just before introducing the anesthetic (time, -30 ), and then again immediately before intraperitoneal injection of glucose $(2 \mathrm{~g} / \mathrm{kg})$ (time 0$)$. The animals were kept sedated by anesthetic supplementation every 45 minutes. Open and closed symbols represent controls and MIRKO mice, respectively. The bars (right) show the calculated area under the curve (minus basal) using the time 0 measurements as base lines. Data are means \pm SEM. $n=$ 7-8 per group. ${ }^{*} P<0.005$ compared with control.

whereas insulin-induced phosphotyrosine associated (PY-associated) PI 3-kinase activity is increased in rat muscle after treadmill exercise (20). In humans and rodents, exercise 3-4 hours before insulin stimulation results in increased insulin sensitivity for glucose transport and Glut 4 translocation, but insulin signaling from the insulin receptor (IR) to glycogen synthase kinase-3 (GSK-3) is not enhanced (21-23; J.F.P. Wojtaszewski, L.J. Goodyear, and E.A. Richter, unpublished data). These observations raise the question of whether IR-mediated signaling is involved in the effect of exercise to increase insulin action.

Recently, muscle-specific insulin receptor knockout (MIRKO) mice were developed using the Cre-loxP system (24). IR content is reduced by more than $95 \%$ as assessed by Western blotting of skeletal muscle homogenates and is not detectable in muscle fibers by immunohistochemical staining in skeletal and heart muscle. Skeletal muscles from MIRKO mice are deficient in insulin signaling at the level of IR and IRS-1 tyrosine phosphorylation in vivo, and insulin does not significantly increase glucose transport in isolated skeletal muscle from these mice (24). In the present study, we have used the MIRKO mice to determine whether normal IR signaling is necessary for the effects of exercise in vivo to increase glucose uptake and glycogen synthesis and to understand whether IR signaling mediates the exercise-induced enhancement of insulin action in skeletal muscle.

\section{Methods}

Animals. The MIRKO mice were generated using the Cre-loxP system as described previously (24), and IR $(l o x / l o x)$ littermates were used as controls. Genotyping was performed by PCR using genomic DNA isolated from the tip of the tail of 3- to 4-week-old mice as described previously (24), and male mice were studied at 9-10 weeks of age. Animals were housed under a 12hour light/dark cycle, having free access to standard laboratory chow and water. All protocols for animal use and euthanasia were approved by the Institutional Animal Care and Use Committee of the Joslin Diabetes Center and were in accordance with National Institutes of Health guidelines.

2-Deoxy-glucose uptake in skeletal muscle. All mice were accustomed to the rodent treadmill (Quinton Instruments Co., Seattle, Washington, USA) by walking or running for $5 \mathrm{~min} / \mathrm{d}$ for 3 consecutive days before the experiment. The animals were fasted from 2300 hours the day before an experiment, and on the experimental day, animals were randomly divided into sedentary and exercised groups. For exercise treatment, mice ran on the treadmill for 1 hour at a speed of $22 \mathrm{~m} / \mathrm{min}$ up a $10 \%$ incline. The animals were sacrificed by decapitation; trunk blood was collected; and soleus muscles were carefully dissected. The muscles were incubated in media consisting of Krebs Ringer bicarbonate buffer supplemented with $8 \mathrm{mM}$ D-mannitol for the first 20 minutes, and uptake of 2-deoxy-glucose (2DG) was then measured for 10 minutes at $30^{\circ} \mathrm{C}$, as described previously (24). For insulin stimulation, pork insulin (Lilly Research Laboratories, Indianapolis, Indiana, USA) was used at a concentration of $5,000 \mu \mathrm{U} / \mathrm{mL}$ and was present throughout all incubations.

Effects of in vivo insulin and exercise treatments on insulin signaling and glycogen metabolism. Overnight-fasted (2300-0800 hours) mice were rested or exercised on a treadmill for 1 hour as already described here. Immediately after the exercise or rest, the animals were injected intraperitoneally with insulin $(0.15 \mathrm{U} / \mathrm{g}$ body weight) or an equal volume of saline. After 5 minutes, the animals were sacrificed by decapitation and trunk blood was collected for blood glucose and plasma insulin determinations. The quadriceps and gastrocnemius muscles were quickly dissected and frozen in liquid nitrogen. The 4 muscles were weighed, finely pulverized, mixed together, and stored at $-80^{\circ} \mathrm{C}$. This mixed muscle preparation was used for signaling studies and measurements of glycogen content and glycogen synthase activity.

\section{Table 1}

Effects of insulin and exercise on blood glucose concentrations

\begin{tabular}{lcc}
\hline Treatment & Control & MIRKO \\
Rest & $81 \pm 5$ & $82 \pm 7$ \\
Insulin & $58 \pm 2^{\mathrm{A}, \mathrm{B}}$ & $74 \pm 3$ \\
Exercise & $71 \pm 7^{\mathrm{B}}$ & $49 \pm 5^{\mathrm{A}}$ \\
Exercise + Insulin & $44 \pm 2^{\mathrm{A}}$ & $47 \pm 3^{\mathrm{A}}$
\end{tabular}

Blood glucose measured after an overnight fast (2300-0800 hours), at rest, after 5 minutes of maximal insulin treatment, and after 1 hour of treadmill exercise followed by either saline or insulin treatment for 5 minutes. Data are given as $\mathrm{mg} / \mathrm{dL}$ (means \pm SEM); $n=6-8$ per group. ${ }^{A} P<0.001$ compared with Rest. ${ }^{\mathrm{B} P}<0.01$ compared with MIRKO. 


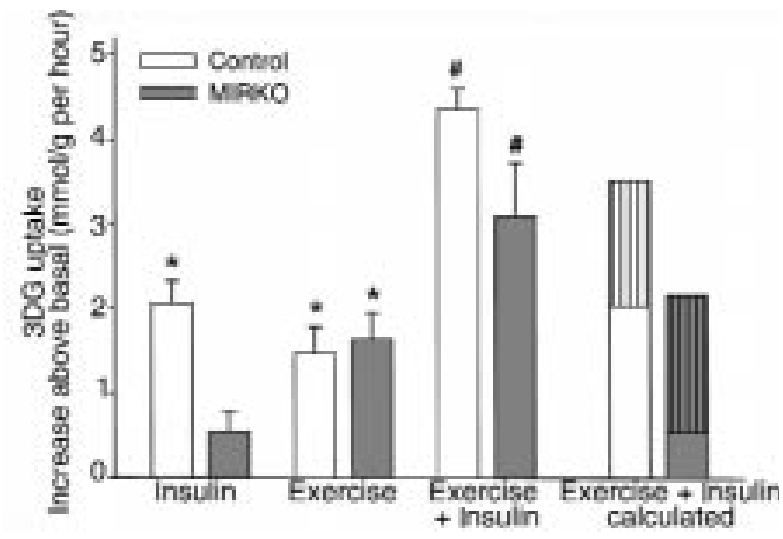

Figure 2

Muscle glucose transport in vitro. 2DG uptake was measured in soleus muscles. 2DG uptake is given as the increase above basal (control $=1.37 \pm 0.04 ;$ MIRKO $=1.86 \pm 0.36[\mu \mathrm{mol} / \mathrm{g}$ per hour $])$. "Exercise + Insulin calculated" represents the expected increase (by calculation) in 2DG uptake during combined stimulation, based on the effect of the individual stimuli. Data are means $\pm \operatorname{SEM}(n=6$ per group). ${ }^{*} P<0.001$ compared with zero. $\# P<0.001$ compared with Insulin or Exercise.

Effects of insulin and muscle contraction in situ on glycogen metabolism. Mice were fasted overnight (2300-0800 hours). Thirty minutes before study, animals were anesthetized with an intraperitoneal injection of pentobarbital ( $90 \mathrm{mg} / \mathrm{kg}$ body weight). During the last $10 \mathrm{~min}$ utes of this period, the sciatic nerve of 1 leg was dissected free and connected to subminiature electrodes (Harvard Apparatus, South Natick, Massachusetts, USA). A pin under the patella tendon fixed the knee of this leg, and a 50-g weight was attached by a wire around the Achilles tendon. The sciatic nerve was stimulated with supraphysiological trains $(5 \mathrm{~V}, 250 \mathrm{~ms})$ of impulses $(0.2 \mathrm{~ms}$, $100 \mathrm{~Hz}$ ) delivered every second for 15 minutes by a Grass S88 stimulator (Grass Instruments, Quincy, Massachusetts, USA). At the end of the 15-minute contraction period, animals were injected intraperitoneally with insulin $(0.15 \mathrm{U} / \mathrm{g}$ body weight $)$ or an equal volume of saline and were sacrificed by decapitation 15 minutes later. The gastrocnemius muscles from both legs were dissected and handled as already described here.

Muscle preparation and signaling assays. Pulverized muscles were homogenized 1:8 (wt/vol) in either buffer A (50 mM HEPES, $150 \mathrm{mM} \mathrm{NaCl}, 20$ $\mathrm{mM} \mathrm{Na} \mathrm{P}_{2} \mathrm{O}_{7}, 20 \mathrm{mM} \beta$-glycerolphosphate, $10 \mathrm{mM} \mathrm{NaF}, 2 \mathrm{mM} \mathrm{Na}_{3} \mathrm{VO}_{4}, 2 \mathrm{mM}$ EDTA, 1\% NP-40, 10\% glycerol, 2 mM PMSF, $1 \mathrm{mM}$ $\mathrm{MgCl}_{2}, 1 \mathrm{mM} \mathrm{CaCl}, 10 \mu \mathrm{g} / \mathrm{mL}$ leupeptin, 10 $\mu \mathrm{g} / \mathrm{mL}$ aprotinin, $3 \mathrm{mM}$ benzamidine $[\mathrm{pH}$ 7.5]) or buffer B (20 mM HEPES, $50 \mathrm{mM} \beta$ glycerolphosphate, $1 \mathrm{mM} \mathrm{Na} \mathrm{VO}_{4}, 2 \mathrm{mM}$ EGTA, 1\% Triton X-100, 10\% glycerol, $1 \mathrm{mM}$ DTT, 1 mM PMSF, $10 \mu \mathrm{M}$ leupeptin, 10 $\mu \mathrm{g} / \mathrm{mL}$ aprotinin, $3 \mathrm{mM}$ benzamidine, $5 \mu \mathrm{M}$ pepstatin A, $0.2 \mathrm{mg} / \mathrm{mL}$ soybean trypsin inhibitor [ $\mathrm{pH} 7.5])$ and rotated end over end

Table 2 for 1 hour at $4^{\circ} \mathrm{C}$. Homogenates were centrifuged at $15,000 \mathrm{~g}$ for 1 hour, the supernatants were collected, and aliquots were stored at $-80^{\circ} \mathrm{C}$. Protein concentrations were determined by the Bradford technique using BSA as standard (25).

For IR tyrosine phosphorylation, aliquots of muscle protein (1 mg, buffer B) were immunoprecipitated overnight with rabbit anti-COOH-terminal IR serum (24) and bound to protein A Sepharose. Proteins were separated by SDS-PAGE, transferred to a nitrocellulose membrane, and immunoblotted for PY (4G10; Upstate Biotechnology Inc., Lake Placid, New York, USA) as described previously (24). Antibody binding was detected using ${ }^{125} \mathrm{I}$ protein $\mathrm{A}$ and quantified using a phosphoimager (Molecular Dynamics, Sunnyvale, California, USA).

For PI 3-kinase activity assays, aliquots of muscle protein (1 mg, buffer A) were immunoprecipitated with either a rabbit anti-IR substrate-1 (IRS-1) antibody (polyclonal antiserum made against a GST fusion peptide of the 511-589 amino acid residues of the mouse IRS-1) or a mouse anti-PY antibody (PY99; Santa Cruz Biotechnology, Santa Cruz, California, USA) and bound to protein A Sepharose. PI 3-kinase activity associated with these immune complexes was assayed as described previously (26). For Akt activity, aliquots of muscle protein (1 mg, buffer A) were immunoprecipitated with anti-Akt 1 antibody (Upstate Biotechnology Inc.), which recognizes both Akt 1 and Akt2. Akt activity in immune complexes was measured with a filter paper (P-81) assay using a GSK-3 peptide as substrate (27).

Akt Ser ${ }^{473}$ and GSK-3 Ser ${ }^{21}$ phosphorylation was assessed by immunoblotting. Protein ( $80 \mu \mathrm{g}$, buffer A) was separated by SDS-PAGE, transferred to a nitrocellulose membrane, blocked in buffer containing 5\% nonfat milk, and incubated with antibody specific for Akt phosphorylated on Ser ${ }^{473}$ (New England Biolabs Inc., Beverly, Massachusetts, USA) or for GSK-3 $\alpha$ phosphorylated on $\operatorname{Ser}^{21}$ (Upstate Biotechnology Inc.). Antibody binding was viewed by enhanced chemiluminescence (Amersham, Arlington Heights, Illinois, USA), and quantified by densitometry (Molecular Dynamics).

Glycogen synthase activity was measured as described previously (28) in the absence or presence of $8 \mathrm{mM}$ glucose 6-phosphate (G6P). Muscle glycogen

IR tyrosine phosphorylation and IRS-1-associated PI 3-kinase activity

\begin{tabular}{lcccc}
\hline Treatment & \multicolumn{2}{c}{$\begin{array}{c}\text { IR tyrosine } \\
\text { phosphorylation }\end{array}$} & \multicolumn{2}{c}{$\begin{array}{c}\text { IRS-1-associated PI 3- } \\
\text { kinase activity }\end{array}$} \\
& Control & MIRKO & Control & MIRKO \\
Rest & $0.21 \pm 0.05$ & $0.14 \pm 0.02$ & $3.7 \pm 0.5$ & $2.7 \pm 0.9$ \\
Insulin & $4.7 \pm 0.2^{\mathrm{A}}$ & $0.29 \pm 0.06$ & $58 \pm 6^{\mathrm{A}}$ & $4.2 \pm 0.9$ \\
Exercise & $0.14 \pm 0.03$ & $0.19 \pm 0.07$ & $2.5 \pm 1.0$ & $2.2 \pm 0.7$ \\
Exercise + Insulin & $4.7 \pm 0.1^{\mathrm{A}}$ & $0.26 \pm 0.06$ & $51 \pm 12^{\mathrm{A}}$ & $4.3 \pm 1.1$
\end{tabular}

Data are expressed in arbitrary units (means \pm SEM). $n=6-8$ per group. ${ }^{A} P<0.001$ com pared with Rest. 
a
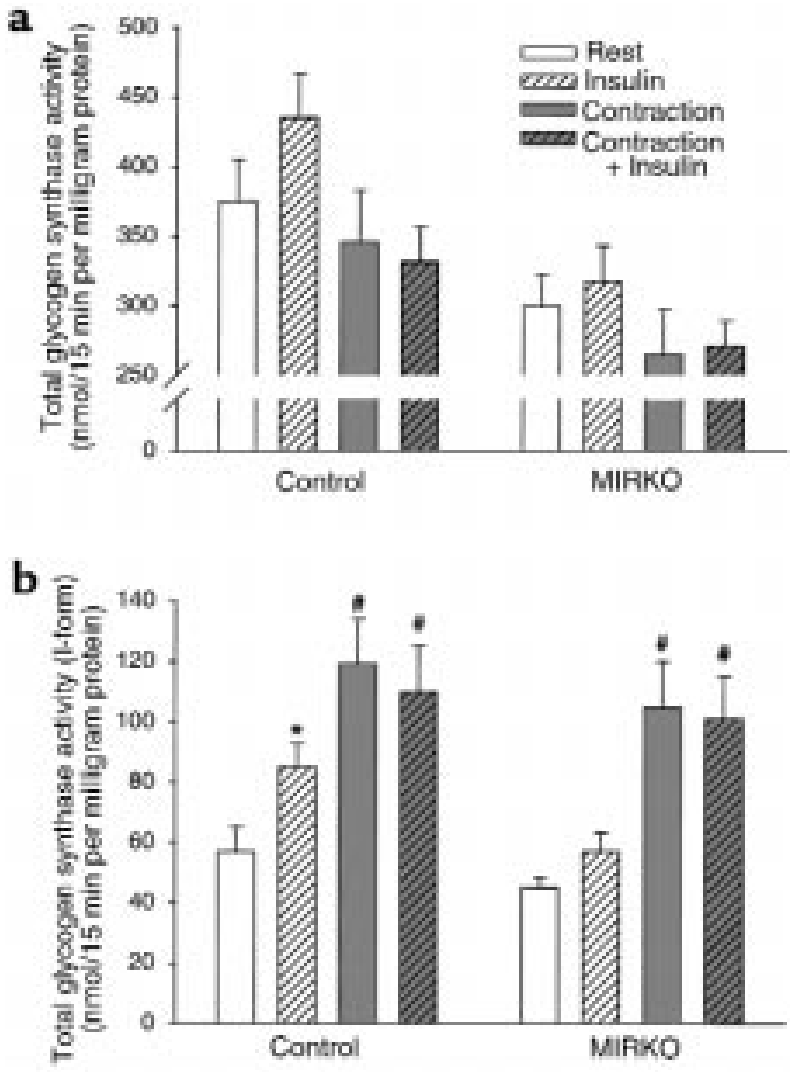

Figure 3

Glycogen synthase activity. Glycogen synthase activity was measured in gastrocnemius muscle from anesthetized animals at rest or after either 15 minutes of contraction in situ, 15 minutes of insulin stimulation, or both. (a) Total glycogen synthase activity in the presence of glucose-6-phosphate $(8 \mathrm{mM})$. (b) Glycogen synthase activity in the absence of glucose-6-phosphate (I-form). Data are means \pm SEM. $n=7-8$ per group. ${ }^{*} P<0.05$ compared with Rest. $\# P<0.01$ compared with Insulin and Rest. ANOVA analysis reveals that total glycogen synthase activity decreases with exercise independent of the presence of insulin in both groups $(P<0.03)$. In addition, total glycogen synthase activity was lower in MIRKO mice compared with control mice $(P<0.0001)$.

content was measured as glycosyl units after hydrolysis as described elsewhere (29).

Glucose tolerance tests. Overnight fasted (1700-0800 hours) mice were anesthetized with pentobarbital (90 $\mathrm{mg} / \mathrm{kg}$ body weight, intraperitoneally), and placed on heating pads maintained at $37^{\circ} \mathrm{C}$. After 30 minutes of anesthesia, the mice were injected with glucose $(2 \mathrm{~g} / \mathrm{kg}$ body weight, intraperitoneally). Tail blood was collected at $-30,-1,15,30,60,90,120,150$, and 180 minutes after glucose injection. All animals received one-fourth the original pentobarbital dose every 45 minutes. Blood glucose concentrations were measured using a glucose meter (One Touch II; Lifescan, Milpitas, California, USA), and plasma insulin using a rat RIA kit (Linco Research Inc., St. Charles, Missouri, USA).

Calculations and statistical analysis. Standards were included in all kinase assays and immunoblots, and interassay variation was accounted for by normalizing data to these control samples. Data are expressed as means \pm SEM. Sta- tistical evaluation was done by unpaired Student's $t$ test or 1- and 2-way ANOVA. When ANOVA revealed significant differences, further analysis was performed using Student's $t$ tests corrected for multiple comparisons (Bonferroni). Differences between groups were considered statistically significant if $P<0.05$.

\section{Results}

Animal characteristics, blood glucose, and plasma insulin concentrations. Compared with the IR (lox/lox) control animals, male MIRKO mice (8-10 weeks old) had slightly lower body weights $(29 \pm 0.4$ vs. $26 \pm 0.5$ g, respectively; $n=37-42 ; P<0.001)$. This was due to a slightly reduced skeletal muscle mass (soleus and quadriceps-gastrocnemius were reduced by $\sim 15-30 \%$ ) and heart size (reduced by $\sim 30 \%$ ). After a 9 -hour fast (2300-0800 hours), plasma insulin concentrations were similar in control and MIRKO mice (18 \pm 1 vs. $19 \pm 4 \mu \mathrm{U} / \mathrm{mL}$, respectively; $n=$ 8). Basal blood glucose concentrations were similar in control and MIRKO mice, but 5 minutes after insulin injections, glucose concentrations were significantly decreased only in control mice (Table 1). When placed on a treadmill at $22 \mathrm{~m} / \mathrm{min}$ (10\% incline) for 1 hour, MIRKO mice were able to perform exercise in a manner indistinguishable from controls. Plasma insulin concentrations decreased by $40-50 \%$ in response to exercise in both strains and increased similarly to supraphysiological levels of approximately $40 \mathrm{mU} / \mathrm{mL} 5$ minutes after insulin injections in both rested and exercised animals. In contrast to controls, in which exercise had little effect on blood glucose, in the MIRKO mice, 1 hour of treadmill exercise decreased blood glucose concentrations by $40 \%(P<0.001)$ (Table 1$)$. When insulin was injected after exercise, blood glucose concentrations did not decrease further in MIRKO mice, whereas there was a significant decrease in the control mice. In anesthetized mice, blood glucose concentrations were not different in control and MIRKO mice before insulin injection $(\sim 92 \mathrm{mg} / \mathrm{dL})$; however, 15 minutes after insulin injection, anesthetized control mice had significantly lower blood glucose concentrations compared with MIRKO mice $(69 \pm 1$ vs. $80 \pm 2 \mathrm{mg} / \mathrm{dL} ; n=7-8$, respectively; $P<0.004)$. These data demonstrate that exogenous insulin has a greater blood glucose-lowering effect in control compared with MIRKO mice, suggesting insulin resistance in MIRKO mice, whereas exercise only lowered glucose concentrations in MIRKO mice.

Muscle glycogen content. After a 9-hour fast, glycogen content was not different in hind-limb muscles from control and MIRKO mice $(178 \pm 9$ vs. $205 \pm 14$ $\mathrm{nmol} / \mathrm{mg}$ protein, respectively; $n=7$ per group). One hour of treadmill exercise decreased muscle glycogen content by $50-60 \%$ in both control and MIRKO mice (94 \pm 13 vs. $86 \pm 21 \mathrm{nmol} / \mathrm{mg}$ protein, respectively; $n=6$ per group). In anesthetized animals, muscle glycogen was not different in control and MIRKO mice at rest (193 \pm 14 vs. $193 \pm 20 \mathrm{nmol} / \mathrm{mg}$ protein, respectively; $n=5$ per group) and decreased similarly by $40-50 \%$ after electrical-induced contractions in situ in 


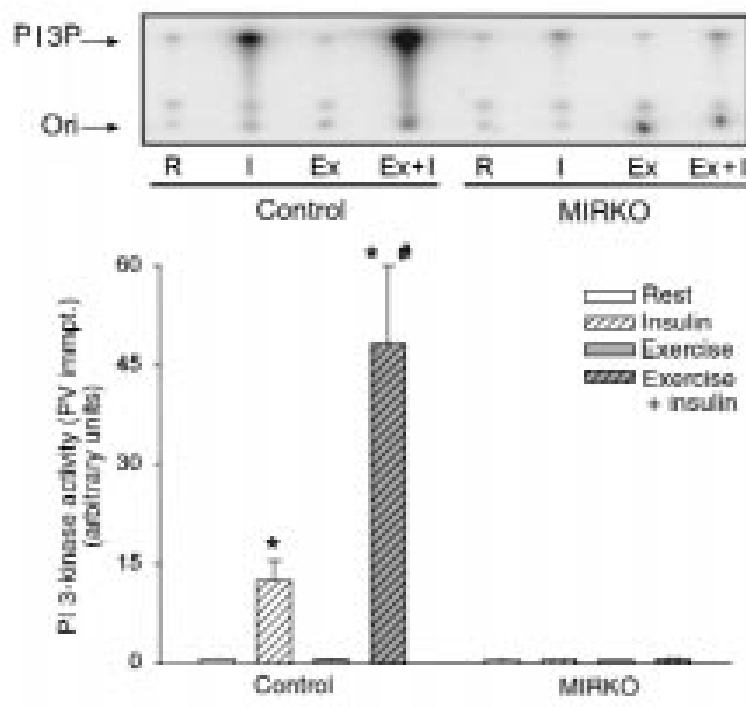

\section{Figure 4}

PY-associated PI 3-kinase activity. Tyrosine-phosphorylated proteins were immunoprecipitated (immpt.)using an anti-PY antibody (PY 99). PI 3-kinase activity was assayed in vitro using PI as substrate. Top panel shows a phosphorimage of a representative TLC plate. Quantified data from multiple experiments are given in the graph below. Data are means \pm SEM. $n=6-8$ per group. ${ }^{*} P<0.001 \mathrm{com}$ pared with Rest. $\# P<0.005$ compared with Insulin.

muscles from both genotypes $(P<0.001)$. Therefore, despite the lack of muscle IRs, MIRKO mice were able to maintain normal levels of glycogen in skeletal muscle. Furthermore, these mice have a normal activation of glycogenolysis in response to contractile activity performed both in vitro and in vivo.

Glucose tolerance. In our previous work, MIRKO mice studied in the awake state had normal glucose tolerance (24). Because mice normally have a high degree of spontaneous physical activity (which can contribute to glucose clearance), in the current study we performed glucose tolerance tests in anesthetized mice after an overnight fast (1700-0800 hours). Basal blood glucose concentrations were similarly increased by 20-35 $\mathrm{mg} / \mathrm{dL}(\sim 40-50 \%) 30$ minutes after injection of the anesthetic (pentobarbital) in both control and MIRKO mice (Figure 1). The MIRKO mice had greater glucose responses to the glucose challenge and, expressed as area under the curve, had an approximately 50\% ( $P<$ 0.005 ) reduction in glucose tolerance compared with the control mice. Thus, disruption of muscle IR expression results in a mild alteration in glucose homeostasis that can be detected in the anesthetized animal but that is hard to detect in awake, freely moving animals.

$2 D G$ uptake in skeletal muscle. To study the effects of exercise in vivo and insulin in vitro on muscle glucose uptake, mice performed treadmill exercise for 1 hour or remained sedentary. The mice were sacrificed immediately after the exercise bout, and soleus muscles were incubated in the absence or presence of insulin. Basal 2DG uptake was not significantly different between muscles from control and MIRKO mice $(1.37 \pm 0.04$ vs.
$1.86 \pm 0.36 \mu \mathrm{mol} / \mathrm{g}$ per hour, respectively; $n=6$ per group). In response to in vitro insulin stimulation, glucose uptake increased significantly above basal in muscle from control mice but not from MIRKO mice (Figure 2), as observed previously (24). Exercise increased 2DG uptake similarly in muscles from both control and MIRKO mice. When mice were first exercised and then stimulated with insulin in vitro, the increase in 2DG uptake was greater than would be predicted from the effects of the 2 stimuli alone. This synergistic effect on 2DG uptake was observed in both control and MIRKO mice (Figure 2). These results demonstrate that exercise in vivo can increase basal and insulin-stimulated glucose uptake in skeletal muscle even in the absence (or near absence) of muscle IRs, suggesting that this phenomenon is unrelated to the actions of insulin through the muscle IR or can occur even with only a very small number of receptors.

Muscle glycogen synthase activity. To study the effects of insulin and exercise on glycogen synthase activity in mice, we anesthetized the animals for 30 minutes before and during treatment, as awake mice have a high basal activity ratio (percent I-form) of muscle glycogen synthase, and we used a muscle contraction model by applying electrical stimulation to the sciatic nerve (15 minutes) to 1 leg of each animal to mimic exercise. This was followed by an intraperitoneal injection of saline or insulin and dissection of muscles 15 minutes later. Surprisingly, the activity of glycogen synthase in the presence of glucose 6-phosphate (total activity) was lower after contraction and tended to be higher after insulin stimulation in mice of both genotypes (Figure 3a). In
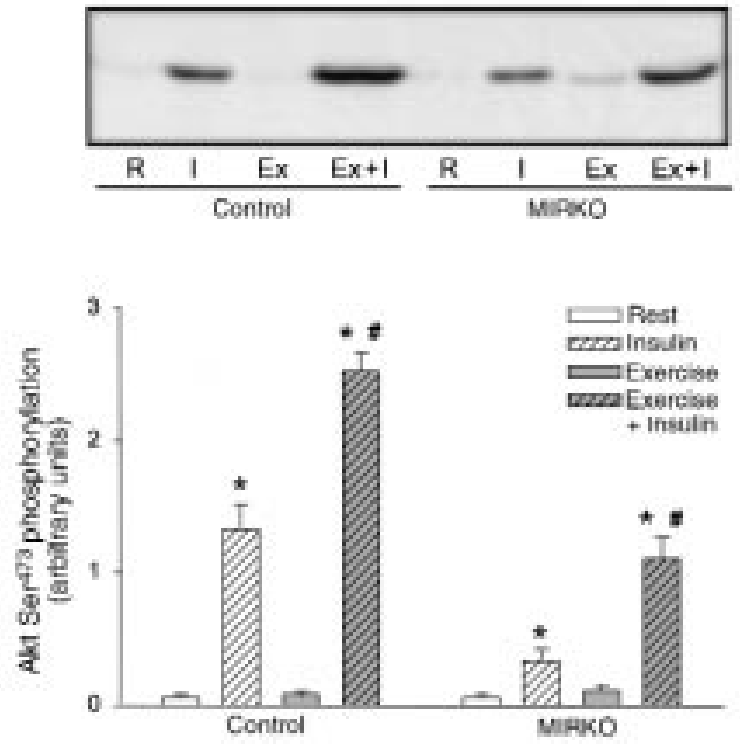

Figure 5

Akt Ser ${ }^{473}$ phosphorylation. Akt Ser ${ }^{473}$ phosphorylation was identified by immunoblotting using a phospho-specific antibody after SDS-PAGE. Top panel shows a representative blot, and the quantified data from multiple experiments are given in the graph below. Data are means \pm SEM. $n=6-8$ per group. ${ }^{*} P<0.001$ compared with Rest. $\# P<0.001$ compared with Insulin. 


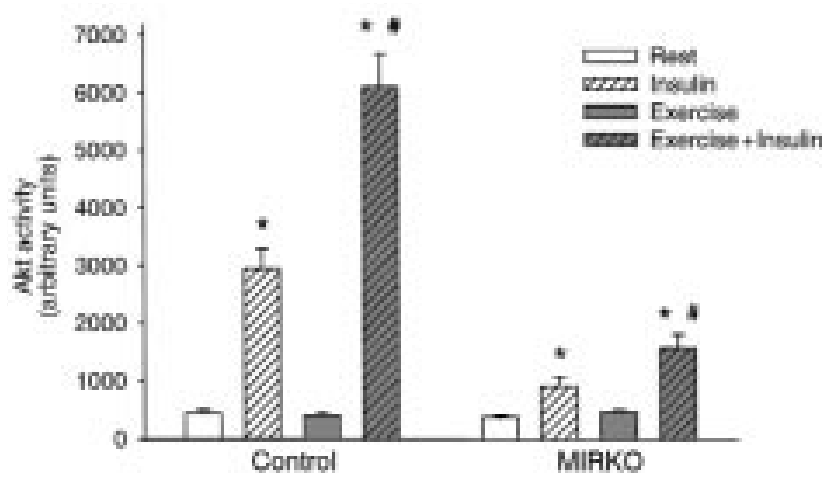

Figure 6

Akt activity. Data are means \pm SEM. $n=6-8$ per group. ${ }^{*} P<0.006$ compared with Rest. $\# P<0.001$ compared with Insulin.

addition, total glycogen synthase activity was significantly lower in muscle from MIRKO mice compared with control mice. The basal glucose 6-phosphate independent glycogen synthase activity (I-form) was similar in skeletal muscle from both genotypes (Figure 3b). Insulin injection significantly increased glycogen synthase activity in skeletal muscle only from control mice. Contraction in situ increased glycogen synthase activity independent of genotype, and the degree of activation by contraction was higher than that reached with maximal insulin stimulation. Compared with contraction alone, there was no additional activation of glycogen synthase when contraction in situ was combined with insulin stimulation in mice of either genotype. Taken together, these data suggest that skeletal muscles from MIRKO mice are insulin resistant for activation of glycogen synthase in vivo, whereas the activation of glycogen synthase with muscle contraction can occur in the absence of muscle IRs. The lack of an additive effect of contraction and insulin on glycogen synthase activation suggests that these stimuli activate the enzyme in part by a similar mechanism, consistent with our recent finding in rat skeletal muscle (30).

Skeletal muscle insulin signaling. To study the effects of exercise and insulin on insulin-signaling molecules, animals were exercised on a treadmill for 1 hour or remained sedentary and were then injected with insulin or saline 5 minutes before they were sacrificed. In mixed hind-limb skeletal muscle from control mice, insulin increased tyrosine phosphorylation of the IR by more than 20 -fold above rested, nonstimulated animals and caused a 20- and 35-fold increase in IRS-1and PY-associated PI 3-kinase activity, respectively (Table 2 and Figure 4). As we have demonstrated previously in rats (19), exercise per se did not change either basal or insulin-stimulated tyrosine phosphorylation of the IR- or IRS-1-associated PI 3-kinase activity. However, prior exercise did result in a 4-fold increase in insulin-stimulated PY- associated PI 3-kinase. By contrast, there was no measurable change in IR tyrosine phosphorylation in MIRKO mice with any of the treatments, and PI 3-kinase activity in PY or IRS-1 immuno- complexes was barely detectable (Table 2 and Figure 4). Insulin treatment lead to a marked Ser ${ }^{473}$ phosphorylation and activation of Akt in control mice, and in line with the upstream PI 3-kinase signaling, exercise potently increased insulin's stimulatory effect on Akt (Figures 5 and 6). Interestingly and in contrast to the inability of insulin to significantly increase glucose transport and proximal IR signaling molecules, Akt phosphorylation and activity were significantly increased with insulin in MIRKO mice (Figures 5 and 6). These effects of insulin were lower compared with control mice for both Akt phosphorylation (6- and 22fold above basal, respectively) and activity (2.4- and 6.5fold above basal, respectively), but, interestingly, these effects were also enhanced (2- to 3 -fold) in exercised muscles. Exercise alone did not increase Akt phosphorylation or activity in either control or MIRKO mice.

In rat skeletal muscle, insulin-stimulated GSK-3 Ser ${ }^{21}$ phosphorylation highly correlates with the degree of deactivation of the $\alpha$ isoform of GSK-3 $\left(r^{2}=0.49, n=\right.$ $18, P<0.001)(30)$. Because Akt is a major GSK-3 kinase expressed in skeletal muscle (31), we would expect GSK-3 to be phosphorylated in a manner resembling the activity of Akt. Five minutes after insulin injection, GSK-3 Ser ${ }^{21}$ phosphorylation was significantly increased in muscles from both control and MIRKO mice, although stimulation in the MIRKO mice was only $50 \%$ of that achieved in control mice (Figure 7). Prior exercise further increased insulin-induced GSK-3 $\mathrm{Ser}^{21}$ phosphorylation in both control and MIRKO mice. In the MIRKO mice, this resulted in a similar level of GSK-3 Ser ${ }^{21}$ phosphorylation as observed in rested control muscle when stimulated by insulin. This effect of exercise was not mediated by exercise per se, as GSK$3 \mathrm{Ser}^{21}$ phosphorylation did not significantly change in either control or MIRKO mice after exercise. Thus, immediately after exercise the maximal insulin activation of PY-associated PI 3-kinase, Akt phosphorylation and activity, as well as phosphorylation of GSK-3, are potently enhanced in skeletal muscle.

\section{Discussion}

It is well established that contraction of skeletal muscle in vitro increases glucose transport in the absence of insulin (5-7). In contrast, it is not clear whether the effects of exercise in vivo to increase muscle glucose uptake can also occur in the absence of insulin or IRmediated cell signaling. Indeed, in the dog, the effect of exercise on glucose uptake is markedly reduced during insulinopenia compared with normoinsulinemia (10). The current study shows that in the mouse, exercise can normally increase muscle glucose transport in the absence or near absence of muscle IRs, supporting the hypothesis of an insulin-independent mechanism for exercise in vivo to increase muscle glucose transport. This also suggests that low circulating levels of insulin are necessary for normal exercise responses in vivo, but this requirement may be due to insulin's acting on cells other than the skeletal muscle fibers. 
A second aspect of this study is the synergistic activation of glucose transport in muscle from control mice when exercise in vivo is followed by insulin stimulation in vitro $(8-13,15,16)$. This was also observed in muscle from MIRKO mice, suggesting that the phenomenon is independent of muscle IRs, and raises the possibility that the synergistic activation of transport is also mediated by insulin's acting on other cell types in the muscle tissue. Alternatively, insulin may bind to receptors other than the IR, leading to activation of alternative signaling molecules that promote glucose transport. One obvious candidate is insulin binding to IGF-1 receptors; however, we believe that this is unlike$l y$, as we fail to see significant receptor tyrosine phosphorylation in MIRKO or control skeletal muscle (24).

In 3T3-L1 adipocytes, the majority of insulin-induced phosphorylated IRS-1 and activated PI 3-kinase is in close association with the cytoskeleton (32) and/or lowdensity microsomes (33), and in both 3T3-L1 adipocytes and L6 muscle cells, an intact actin filament arrangement is necessary for a full response of the glucose transport system to insulin $(34,35)$. Thus, preassembling of signaling molecules on an internal membrane or cytoskeletal scaffold may be necessary to obtain not only specificity, but also responsiveness in a signaling cascade (32). We speculate that exercise induces a relocalization of insulin-signaling molecules (PI 3-kinase, Akt, GSK3) in such a way that more substrates are accessible for activation. This could increase responsiveness for activation by insulin, without showing any activation of these signaling molecules during or after exercise in the absence of insulin.

A fascinating finding of the current study is that in the control mice, there was enhanced insulin-induced PY-associated PI 3-kinase activity after exercise that could not be explained by increased IRS-1-associated PI 3 -kinase activity. This is unlikely due to IRS-2 because skeletal muscle expresses very little IRS-2 and because IRS-2 is not tyrosine phosphorylated by exercise per se (27), nor is IRS-2 necessary for the combined effects of exercise plus insulin on glucose transport (36). We have also determined that Gab-1 binding to PI 3-kinase cannot explain the increased PI 3-kinase activity (J.F.P. Wojtaszewski and L.J. Goodyear, unpublished observation). This raises the possibility that there is another tyrosine phosphoprotein that binds and activates PI 3-kinase in the period after exercise. With the use of Western blot analysis of PY proteins in phosphotyrosine immunoprecipitates, we have not identified any major differences in the insulin-induced phosphorylation pattern in rested and exercised muscles from either control or MIRKO mice (data not shown). The elucidation of this protein may provide a critical clue in determining the mechanism by which exercise increases insulin responsiveness and sensitivity in muscle and will be an important area for future investigation.

In the current study, insulin modestly increased Akt activity and GSK-3 serine phosphorylation in the MIRKO mice. There are several possible mechanisms
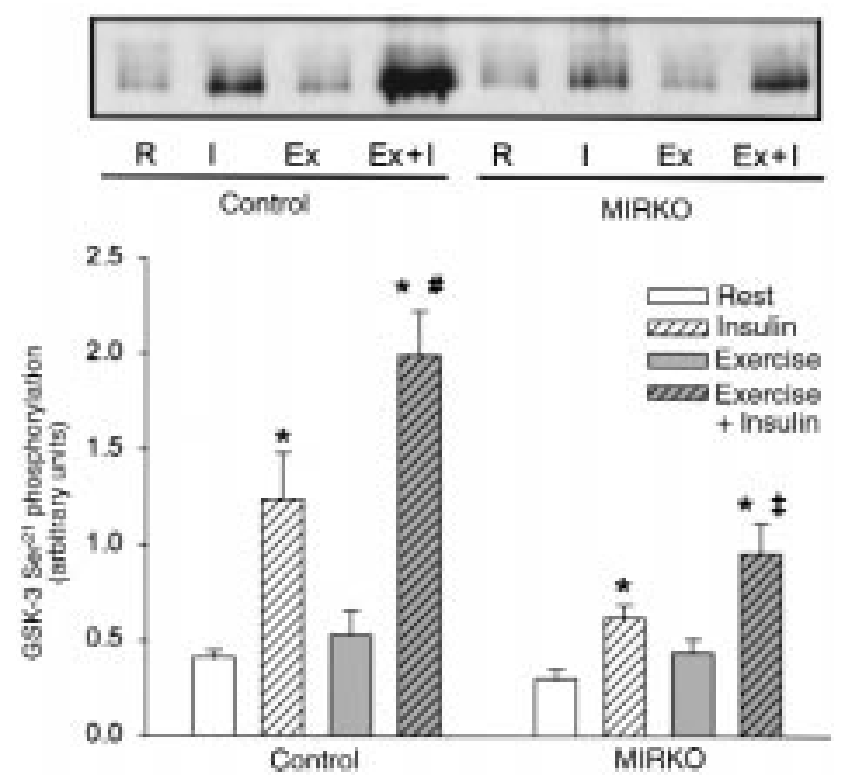

\section{Figure 7}

GSK-3 Ser ${ }^{21}$ phosphorylation. GSK-3 Ser ${ }^{21}$ phosphorylation was identified by immunoblotting using a phospho-specific antibody after SDS-PAGE. Top panel shows a representative blot, and the quantified data from multiple experiments are given in the graph below. Data are means \pm SEM. $n=6-8$ per group. ${ }^{*} P<0.004$ compared with Rest. $\# P=0.05 . \ddagger P=0.08$ compared with Insulin.

that may result in activation of these signaling molecules. First, skeletal muscle is a heterogeneous tissue containing multiple cells of different origin, and therefore it is conceivable that other insulin sensitive cells are partially responsible for the increase in Akt activity and GSK-3 phosphorylation seen in MIRKO muscle. However, we think that it is unlikely that nonmuscular cells could account for up to $40 \%$ of the insulininduced signaling in the muscle tissue and also display an exercise-dependent signaling response. Second, the supraphysiological insulin concentration used in our studies could activate IGF-1 receptors and subsequently result in Akt and GSK-3 activation. On the other hand, as reported previously (24), we did not detect any increase in PY proteins at the molecular weight of the IGF-1 receptor in muscle from either control or MIRKO mice, and we did not observe PI 3kinase activation in MIRKO mice, which we would expect if IGF-1 receptor signaling was a major contributor. Third, it is possible that insulin activates upstream signaling in muscles from MIRKO mice, including IRs, to an extent that is too small to be detected but, by amplification, is sufficient for a significant Akt and GSK-3 activation. Finally, it is possible that insulin can stimulate some alternative, non-PI 3-kinase-dependent pathways to activate Akt and GSK3 in skeletal muscle. In fact, there are examples of wortmannin-insensitive mechanisms to increase Akt activity in the literature $(37,38)$. Regardless of the mechanism of the small increase in Akt and GSK-3 signaling, it is possible that these molecules may mediate 
the tendency for insulin to increase slightly glucose transport and glycogen synthase activity in skeletal muscle from MIRKO mice.

Another surprising feature of the MIRKO mouse is that skeletal muscle has normal concentrations of muscle glycogen, despite a near absence of insulin-stimulated glycogen synthase activity. Physical exercise and contractile activity decreased glycogen levels and activated glycogen synthase normally in MIRKO mice. These data suggest that alternative, insulin-independent mechanisms also contribute to the maintenance of muscle glycogen levels in the MIRKO mice.

In summary, skeletal muscles of MIRKO mice are severely insulin resistant for activation of glucose transport in vitro and glycogen synthase in vivo, and under some conditions, this is manifested by insulin resistance at the whole-body level when challenged with exogenous glucose. However, the ability of exercise in vivo to increase skeletal muscle glucose transport, to increase glycogen synthase activity, and to enhance insulin-stimulated glucose transport can occur in the absence of muscle IRs and thus contribute to the ability of MIRKO mice to maintain normal or near normal glucose tolerance during normal free-living conditions.

\section{Acknowledgments}

This study was supported by grants from the National Institutes of Health (AR42238 and AR45670 to L.J. Goodyear; DK31036 to C.R. Kahn; and DK09817-02 to M.D. Michael). J.F.P. Wojtaszewski was supported by a postdoctoral fellowship from the Alfred Benzon Foundation, Denmark. Y. Higaki was supported by the Ministry of Education, Science, Sports and Culture, Japan.

1. Cheatham, B., and Kahn, C.R. 1995. Insulin action and the insulin signaling network. Endocr. Rev. 16:117-142.

2. Czech, M.P., and Corvera, S. 1999. Signaling mechanisms that regulate glucose transport. J. Biol. Chem. 274:1865-1868.

3. Richter, E.A. 1996. Glucose utilization. In Handbook of physiology. L.B. Rowell and J.T. Shepherd, editors. Oxford University Press. New York, NY. 912-951.

4. Holloszy, J.O., and Hansen, P.A. 1996. Regulation of glucose transport into skeletal muscle. Rev. Physiol. Biochem. Pharmacol. 128:99-193.

5. Ploug, T., Galbo, H., and Richter, E.A. 1984. Increased muscle glucose uptake during contractions: no need for insulin. Am. J. Physiol. 247:E726-E731

6. Wallberg-Henriksson, H., and Holloszy, J.O. 1985. Activation of glucose transport in diabetic muscle: responses to contraction and insulin. Am. J. Physiol. 249:C233-C237.

7. Nesher, R., Karl, I., and Kipnis, D. 1985. Dissociation of effects of insulin and contraction on glucose transport in rat epitrochlearis muscle. Am. J. Physiol. 249:C226-C232.

8. Turinsky, J. 1987. Glucose and amino acid uptake by exercising muscles in vivo: effect of insulin, fiber population, and denervation. Endocrinology. 121:528-535.

9. Vranic, M., Kawamori, R., Pec, S., Kovacevic, N., and Wrenshall, G.A. 1976. The essentiality of insulin and the role of glucagon in regulating glucose utilization and production during strenuous exercise in dogs. $J$. Clin. Invest. 57:245-255.

10. Wasserman, D.H., Mohr, T., Kelly, P., Lacy, B., and Bracy, D. 1992. Impact of insulin deficiency on glucose fluxes and muscle glucose metabolism during exercise. Diabetes. 41:1229-1238.

11. DeFronzo, R., Ferrannini, E., Sato, Y., Felig, P., and Wahren, J. 1981. Synergistic interaction between exercise and insulin on peripheral glucose uptake. J. Clin. Invest. 68:1468-1474.

12. Wasserman, D.H., et al. 1991. Interaction of exercise and insulin action in humans. Am. J. Physiol. 260:E37-E45.

13. Berger, M., Hagg, Goodman, M.N., and Ruderman, N.B. 1975. Glucose metabolism in perfused skeletal muscle. Biochem. J. 146:231-238.

14. Ploug, T., Galbo, H., Vinten, J., Jørgensen, M., and Richter, E.A. 1987. Kinetics of glucose transport in rat muscle: effects of insulin and contractions. Am. J. Physiol. 253:E12-E20.

15. Vergauwen, L., Hespel, P., and Richter, E.A. 1994. Adenosine receptors mediate synergistic stimulation of muscle glucose uptake and transport by insulin and by contractions in rat skeletal muscle. J. Clin. Invest. 93:974-981.

16. Hespel, P., Vergauwen, L., Vandenberghe, K., and Richter, E.A. 1995. Important role of insulin and flow in stimulating glucose uptake in contracting skeletal muscle. Diabetes. 44:210-215.

17. Ivy, J.L., and Kuo, C.H. 1998. Regulation of GLUT4 protein and glycogen synthase during muscle glycogen synthesis after exercise. Acta Physiol. Scand. 162:295-304.

18. Hayashi, H., Wojtaszewski, J.F.P., and Goodyear, L.J. 1997. Exercise regulation of glucose transport in skeletal muscle. Am. J. Physiol. 273:E1039-E1051.

19. Goodyear, L.J., Giorgino, F., Balon, T.W., Condorelli, G., and Smith, R.J. 1995. Effects of contractile activity on tyrosine phosphoproteins and PI 3-kinase in rat skeletal muscle. Am. J. Physiol. 268:E987-E995.

20.Zhou, Q., and Dohm, G. 1997. Treadmill running increases phosphatidylinositol 3-kinase activity in rat skeletal muscle. Biochem. Biophys. Res. Commun. 236:647-650.

21. Richter, E.A., Mikines, K.J., Galbo, H., and Kiens, B. 1989. Effect of exercise on insulin action in human skeletal muscle. J. Appl. Physiol. 66:876-885.

22. Wojtaszewski, J.F.P., Hansen, B.F., Kiens, B., and Richter, E.A. 1997. Insulin signaling in human skeletal muscle: time course and effect of exercise. Diabetes. 46:1775-1781.

23. Hansen, P.A., Nolte, L.A., Chen, M.M., and Holloszy, J.O. 1998. Increased GLUT4 translocation mediates enhanced insulin sensitivity of muscle glucose transport after exercise. J. Appl. Physiol. 85:1218-1222.

24. Brüning, J.C., et al. 1998. A muscle-specific insulin receptor knockout exhibits features of the metabolic syndrome of NIDDM without altering glucose tolerance. Mol. Cell. 2:559-569.

25. Bradford, M.M. 1976. A rapid and sensitive method for the quantitation of microgram quantities of protein utilizing the principle of protein-dye binding. Anal. Biochem. 72:248-254.

26. Wojtaszewski, J.F.P., Hansen, B.F., Ursø, B., and Richter, E.A. 1996. Wortmannin inhibits both insulin- and contraction-stimulated glucose uptake and transport in rat skeletal muscle. J. Appl. Physiol. 81:1501-1509.

27. Sherwood, D., et al. 1999. Differential regulation of MAP kinase, $\mathrm{P} 70^{\mathrm{S} 6 \mathrm{~K}}$, and Akt by contraction and insulin in rat skeletal muscle. Am. J. Physiol. 276:E870-E878

28. Thomas, J.A., Schlender, K.K., and Larner, J. 1968. A rapid filter paper assay for UDP glucose-glycogen glucosyltransferase, including an improved biosynthesis of UDP-14C-glucose. Anal. Biochem. 25:486-499.

29. Lowry, O.H., and Passonneau, J.V. 1972. A flexible system of enzymatic analysis. Academic Press Inc. London, United Kingdom. 291 pp.

30. Markuns, J.F., Wojtaszewski, J.F.P., and Goodyear, L.J. 1999. Insulin and exercise decrease glycogen synthase kinase- 3 activity by different mechanisms in rat skeletal muscle. J. Biol. Chem. 274:24896-24900.

31. Cross, D.A.E., Alessi, D.R., Cohen, P., Andjelkovic, M., and Hemmings, B.A. 1995. Inhibition of glycogen synthase kinase-3 by insulin mediated by protein kinase B. Nature. 378:785-789.

32. Clark, S.F., Martin, S., Carozzi, A.J., Hill, M.M., and James, D.E. 1998. Intracellular localization of phosphatidylinositide 3-kinase and insulin receptor substrate- 1 in adipocytes: potential involvement of a membrane skeleton. J. Cell Biol. 140:1211-1225.

33. Inoue, G., Cheatham, B., Emkey, R., and Kahn, C.R. 1998. Dynamics of insulin signaling in 3T3-L1 adipocytes. J. Biol. Chem. 273:11548-11555.

34. Wang, Q., Bilan, P.J., Tsakiridis, T., Hinek, A., and Klip, A. 1998. Actin filaments participate in the relocalization of phosphatidylinositol 3-kinase to glucose transporter containing compartments and in the stimulation of glucose uptake in 3T3-L1 adipocytes. Biochem. J. 331:917-928.

35. Tsakiridis, T., et al. 1997. Involvement of the actin network in insulin signaling. Soc. Gen. Physiol. Ser. 52:257-271.

36. Higaki, Y., et al. 1999. IRS-2 is not necessary for insulin- and exercise-stimulated glucose transport in skeletal muscle. J. Biol. Chem. 274:20791-20804.

37. Moule, S.K., et al. 1997. Regulation of protein kinase B and glycogen synthase kinase 3 by insulin and beta-adrenergic agonists in rat epididymal fat cells. Activation of protein kinase B by wortmannin-sensitive and insensitive mechanisms. J. Biol. Chem. 272:7713-7719.

38. Sable, C.L., Filippa, N., Hemmings, B., and Van Obberghen, E. 1997. cAMP stimulates protein kinase B in a wortmannin-insensitive manner. FEBS Lett. 9:253-257. 